\title{
Circular RNAs in gastric cancer: Biomarkers for early diagnosis (Review)
}

\author{
CHUN-MEI YANG ${ }^{1,2^{*}}$, GUANG-LEI QIAO $^{1 *}$, LI-NA SONG $^{1}$, SHISAN BAO $^{1,3}$ and LI-JUN MA ${ }^{1}$ \\ ${ }^{1}$ Department of Oncology, Tongren Hospital, Shanghai Jiao Tong University School of Medicine, Shanghai 200336; \\ ${ }^{2}$ Department of Clinical Laboratory Diagnostics, Beihua University, Jilin City, Jilin 132012, P.R. China; \\ ${ }^{3}$ Discipline of Pathology, School of Medical Science and Bosch Institute, Charles Perkins Centre, \\ The University of Sydney, Sydney, NSW 2006, Australia
}

Received November 27, 2019; Accepted April 9, 2020

DOI: $10.3892 / \mathrm{ol} .2020 .11623$

\begin{abstract}
Circular RNAs (circRNAs) are highly conserved and stable closed-loop non-coding RNAs. They are involved in numerous biological functions, including regulating gene transcription or protein translation by interacting with proteins and regulating expression of microRNAs. The aberrant expression of circRNAs has been reported in many cancers, including gastric cancer. By regulating gene expression, circRNAs are able to affect the proliferation, invasion and metastasis of gastric cancer. The current review focused on the characteristics and biological functions of circRNAs, the carcinogenic potential and the possible implications of circRNAs on the diagnosis and treatment of gastric cancer. In conclusion, circRNAs may serve as potential biomarkers for diagnosis, as well as therapeutic targets.
\end{abstract}

\section{Contents}

1. Introduction

2. Biological functions of circRNAs

3. circRNAs regulate the proliferation and progression

4. circRNAs have a potential clinical value in gastric cancer

5. Future prospects

6. Conclusions

Correspondence to: Dr Li-Jun Ma, Department of Oncology, Tongren Hospital, Shanghai Jiao Tong University School of Medicine, 1111 Xianxia Road, Shanghai 200336, P.R. China

E-mail: 1jma56@126.com

Dr Shisan Bao, Discipline of Pathology, School of Medical Science and Bosch Institute, Charles Perkins Centre, The University of Sydney, Johns Hopkins Drive, Camperdown, Sydney, NSW 2006, Australia

E-mail:bob.bao@sydney.edu.au

${ }^{*}$ Contributed equally

Key words: circular RNAs, gastric cancer, novel biomarkers

\section{Introduction}

Gastric cancer is the fifth most common cancer and third leading cause of cancer mortality in the world, according to the International Agency for Research on Cancer in 2018 (1). The morbidity of gastric cancer affected $>1$ million in 2018, accounting for $5.7 \%$ of new cancer cases globally. Worldwide, gastric cancer mortality is $\sim 783,000$, accounting for $8.2 \%$ of the total global deaths caused by cancer in 2018 (1). Diagnosis of gastric cancer relies on endoscopic examination and analyses of serumbiomarkers in the clinical practice (2). However, endoscopy cannot be used for large-scale screening, and detection by serum biomarkers lacks sensitivity and specificity (3). To date, there is no simple and efficient diagnostic method that allows for the early diagnosis of gastric cancer to improve its prognosis.

Circular RNAs (circRNAs) are a group of single-strand closed-loop RNAs that are derived from back-splicing and are widely found in serum and saliva $(4,5)$. circRNAs serve a regulatory role at the transcriptional and/or post-transcriptional level, but some can only function at the transcriptional level. Previous research has found that biological functions of circRNAs include microRNA (miRNA) sponging, regulation of transcription and translation, and interaction with RNA-binding proteins (4,6-8). Additionally, the dysregulation of circRNA expression is suggested to play an important role in the pathogenesis and diagnosis of gastric cancer.

Although little is known about the role of circRNAs in the development of gastric cancer at the molecular level, circRNAs secreted into the extracellular environment can be identified in blood, stool and other body fluids (9-12). Thus, circRNAs might be used as new potential biomarkers for the diagnosis and therapeutic targeting of gastric cancer.

\section{Biological functions of circRNAs}

circRNAs are abnormally expressed in various cancer types, including gastric cancer (13-16), such as circCCDC66, circPIP5K1A, circ-ATAD1 are higher expressed, but circMTO1 expression is lower in gastric cancer (17-20). circRNAs may cause cancer by interacting with tumor-associated miRNAs, proteins and genes, and by participating in pathophysiological activities, including proliferation, invasion 
and apoptosis (21-23). CDR1 antisense RNA (CDR1as) is an abundantly expressed circRNA in the mammalian brain with $>70$ miRNA (miR)-7 binding sites (24). CDRlas/miR-7 is a classical tumor-associated circRNA-miRNA sponge system. CDRlas inhibits miR-7 activity by competing with miR-7, which ultimately upregulates the expression of oncogenes, such as epidermal growth factor receptor, $\mathrm{NF}-\kappa \mathrm{B}$, and eventually promotes the development of cancer (25).

Overexpression of CDR1as inhibits the proliferation and invasion of glioma, breast cancer, gastric cancer and colorectal cancer (26-29). By contrast, upregulation of other circRNAs, such as circHIPK3, hsa_circ_001569 and circSCAF11, promotes the development of bladder and colorectal cancers, as well as glioma (30-32). In addition, abundant exosome circRNAs in the serum differentiate gastric cancer by transmitting a signal to local and remote cells, such as infiltrating immune cells, stromal cells and endothelial cells. These cells are vital components of the tumor microenvironment and enhance tumor growth, angiogenesis and metastasis (33). Such circRNAs may be useful for early and specific diagnosis of tumors.

miRNA sponge. circRNAs can absorb specific miRNAs through numerous miRNA binding sites (Fig. 1A). Conversely, they may serve as highly efficient sponges with miRNA to inhibit the biological functions of miRNAs and prevent them from binding to their target genes (34). For example, Sex-determining region $\mathrm{Y}$, the testis-specific circRNA, inhibits the activity of miR-138 through multiple miR-138 binding sites and potentially regulates expression of miR-138 target genes (35). It is speculated that miRNA sponge effects achieved by circRNA formation are a common phenomenon (35). circ-CEP85L functions as a sponge of miR-942-5p to suppress expression of its target genes NFKBIA (36). In addition, circFGFR4 promotes the differentiation of myoblasts by sponging miR-107 and suppressing its target gene Wnt3 expression (37).

Interaction with RNA-binding proteins (RBPS). circRNAs are involved in numerous physiological processes by interacting with a variety of RBPs to form RNA-protein complexes, regulating gene transcription and influencing the formation of circRNAs (Fig. 1B). RBPs affect the expression of downstream target genes and miRNA sponges by competing with miRNAs $(6,7)$. RBPs also act as trans-factors to regulate circRNA generation. Modulation of splicing factor muscle blind (MBL) expression affects the biosynthesis of circMBL through specific binding to MBL binding sites within introns of circMBL (38). circMBL regulates parental gene expression by binding to its own pre-mRNA flanking introns binding sites at the transcriptional level. circRNAs have a binding site for the enzyme and its substrate, thus it can directly bind to protein or act as a scaffold between two or more proteins. It has been reported that circ-Amotl1 interacts with proto-oncogene c-Myc, STAT3, pyruvate dehydrogenase kinase land AKT1 and affects the expression of its target genes by promoting nuclear translocation of these proteins $(39,40)$. In addition, RBPs are also involved in the post-transcriptional regulation of miRNA expression and interact with miRNA regulators by cooperative and competitive inhibition (41). Pumilioprotein, a cooperative RBP, promotes miRNA binding to target mRNA, which inhibits protein translation by binding to a specific short sequence in the 3'UTR of target mRNA, leading to altered spatial conformation of mRNA (42).

Regulation of transcription. circRNAs derived from exons are located in the cytoplasm and have an open reading frame (ORF) to drive translation (Fig. 1C) (43). In addition, some circRNA protein translation is initiated after being modified by $\mathrm{N}^{6}$-methyladenosine $\left(\mathrm{m}^{6} \mathrm{~A}\right)$, such as circRNAs initiates the protein translation by recruit YTHDF3 and EIf4G2 through the $\mathrm{m}^{6} \mathrm{~A}$ modification site (44). circANRIL regulates the expression of INK4/ARF by affecting the binding of ANRIL to polycomb-group complex and inhibiting the transcription of the coding gene INK4 and its variable reading frame genes (45). circPABPN1 reduces the translational efficiency of PABPN1 mRNA by competitively binding the RBP human antigen $\mathrm{R}$ (HuR) and prevents HuR binding to PABPN1 mRNA (46). In addition, a small amount of circRNAGFP participates in the translation process as a template (47).

Regulation of host gene expression. circRNA sequences are highly similar to their homogenous linear mRNA. circRNAs act as positive regulators of the activity of RNA polymerase II (RNA pol II), affecting expression of parental genes and transcription (Fig. 1D). circEIF3J and circPAIP2, both exon-intron circRNAs (EIciRNAs), interact with U1 small nuclear ribonucleoprotein (snRNP) through RNA-RNA interactions to form EIciRNA-U1 snRNP complexes. This complex interacts with RNA pol II in the promoter region of host genes to cis-regulate the expression of its host genes (48). Additionally, circ-ITCH has common miRNA binding sites with the 3' untranslated region of itchy E3 ubiquitin protein ligase (ITCH). circ-ITCH interacts with miR-7, miR-17 and miR-214 to promote the expression of ITCH (49).

\section{3. circRNAs regulate proliferation and progression in gastric cancer}

circRNAs regulate the proliferation and progression as miRNA sponges in gastric cancer. circRNAs promote malignant cell development, proliferation and metastasis by sponging miRNAs resulting in the dysregulated expression of miRNAs and the disruption of target genes in gastric cancer. Upregulated circPDSS1 expression promotes the expression of NIMA-related kinase 2 (NEK2) in gastric cancer through a miR-186-5p sponge in vivo and in vitro (Fig. 2) (50). Subsequently NEK2, which is associated with cell centrosome and cell cycle in gastric cancer, enhances cell proliferation and cell cycle by regulating cell mitosis in this type of cancer (51). circPVT1, a potential oncogenic gene, inhibits the activity of miR-125 and promotes the proliferation of gastric cancer through c-Myc (52). In addition, circLARP4 is inversely associated with tumor size and lymph node metastasis in gastric cancer by regulating the target gene large tumor suppressor kinase 1 and Yes-associated protein (YAP) of miR-424, resulting in the inhibition of DNA synthesis, cell proliferation and invasion (Fig. 2) (53). circYAP1 inhibits cellular proliferation, invasion and cell cycle by suppressing cyclin dependent kinase inhibitor 1B. Downregulated circYAP1 in gastric cancer is linked to poor prognosis and low 5 year survival rate (Fig. 2) (53). 


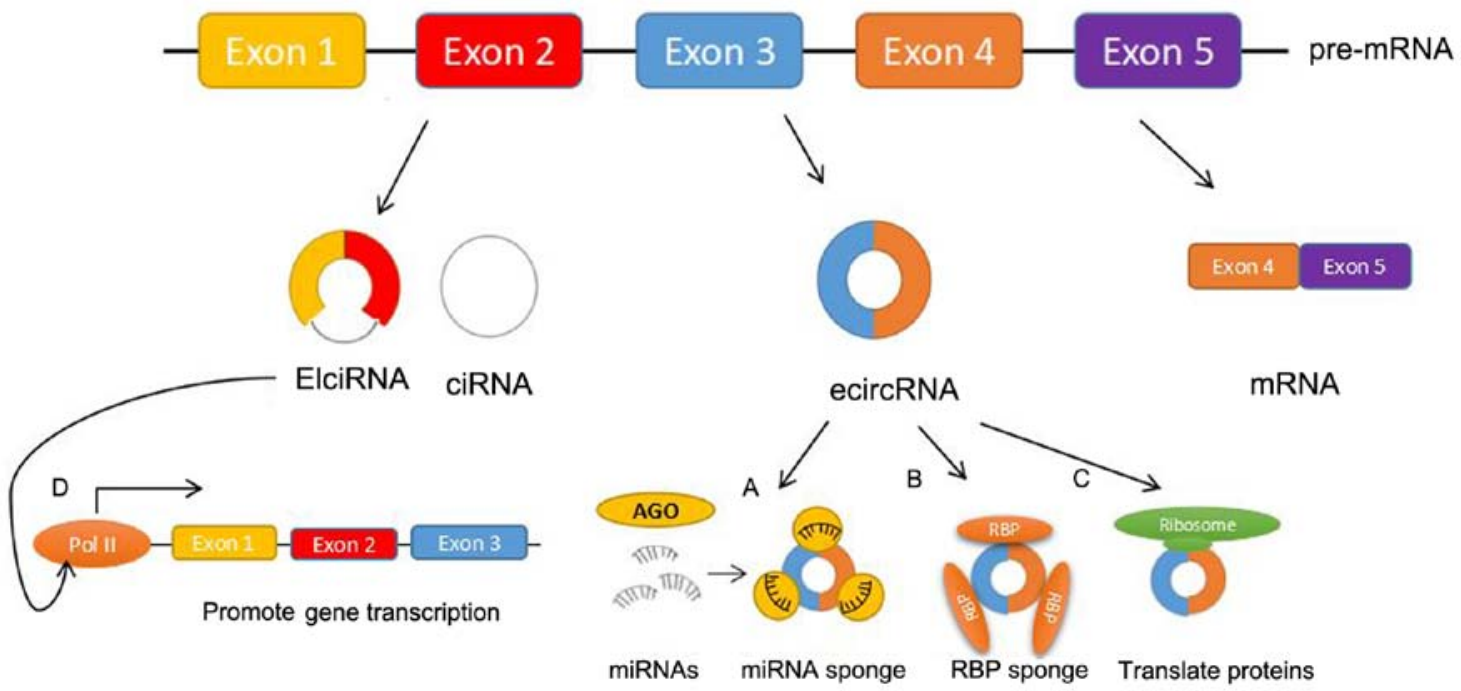

Figure 1. Biological functions of circRNAs. A pre-mRNA is back-spliced, producing three different types of circRNA, including ciRNAs, ecirRNAs and EIciRNA. The biological functions of circRNAs are regulated by different mechanisms. (A) miRNA sponge. circRNAs absorb specific miRNA to regulate the biological function of miRNA, affecting miRNA target gene expression. (B) RBP sponge. circRNAs interact with a variety of RBPs to form RNA-protein complexes, regulating their cellular localization and activity. (C) Translate proteins. Some circRNAs can be translated into proteins that exert specific biological effects and affect tumor progression. (D) Promote gene transcription. ciRNAs and EIciRNAs promote the transcription of their parental genes by acting as positive regulators of Pol II activity. AGO, protein argonaute; circRNAs, circular RNAs; ciRNAs, intronic circular RNAs; ecirRNAs, exonic circular RNAs; EIciRNA, exon-intron circular RNAs; miRNAs, micro RNAs; Pol II, RNA polymerase II; RBPs, RNA binding proteins.

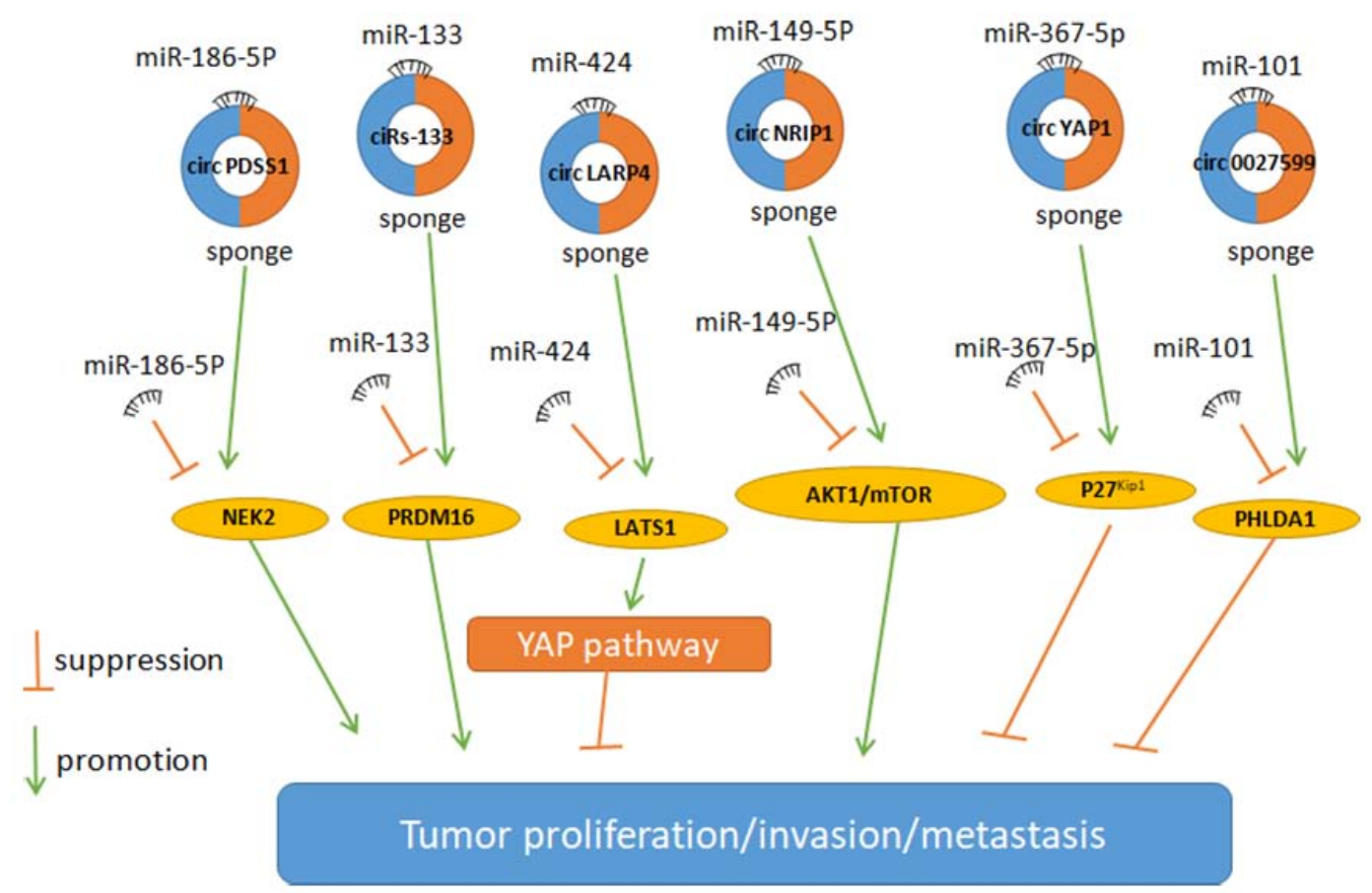

Figure 2. Roles of circRNAs in gastric cancer. circRNAs function as sponges for the indicated miRNAs, which regulate the respective target genes to promote or inhibit tumor proliferation, invasion and metastasis. circRNA, circular RNA; LARP4, La ribonucleoprotein 4; LATS1, large tumor suppressor kinase 1; miR, microRNA; NEK2, NIMA related kinase 2; NRIP1, nuclear receptor interacting protein 1; p $^{\text {kip1 }}$, cyclin dependent kinase inhibitor 1B; PDSS1, decaprenyl diphosphate synthase subunit 1; PHLDA1, pleckstrin homology like domain family A member 1; PRDM16, PR/SET domain 16; YAP1, Yes associated protein 1.

circ_100269 suppresses the p53 signaling pathway by sponging miR-630 to inhibit proliferation and invasion of gastric cancer (54). In addition, circ_0027599 sponges miR-101 to inhibit the migration and invasion of gastric cancer by targeting pleckstrin homology-like domain family A member 1 gene (Fig. 2) (55). These differential expressions of circRNAs in the different aforementioned cancers play crucial but different roles in determining cell fate, inducing tumor-suppressive or oncogenic effects by acting as a sponge to multiple different miRNAs, forming a circRNA-miRNA-mRNA axis (50-55). Thus, circRNAs have the ability to participate in many physiological and pathological processes, suggesting that the regulation of circRNAs could offer a potential therapeutic window for gastric cancer. 
circRNAs regulate the proliferation and progression through the RBP sponge in gastric cancer. circRNAs not only function as miRNA sponges to regulate miRNA expression, but also serve important roles in regulating protein production (56). circRNAs affect the proliferation and metastasis potential of malignant cells, including gastric cancer cells, by directly interacting with proteins or binding to the miRNA-acting factor to regulate specific downstream target genes (57).

Downregulated circPVRL3 also promotes the proliferation of gastric cancer by promoting protein encoding (58). It might play a role in the assembling of RBP complexes by binding to AGO2, FUS RNA-binding protein, lin-28 homolog A, polypyrimidine tract binding protein and eukaryotic translation initiation factor 4A3. In addition, circPVRL3 contains internal ribosome entry site, ORF and $\mathrm{m}^{6} \mathrm{~A}$ modifications, these modifications initiate the protein translation by recruiting ribosomes $(59,60)$. The translational products may exert specific biological effects or interfere with protein-protein interactions to affect tumor progression $(44,61)$. Another study reported that circFAT1(e2), located in cytoplasm and nucleus of gastric cancer cells, is downregulated in gastric cancer (62). circFAT1(e2) directly binds to Y-box binding protein-1 (YBX1) for DNA- and RNA-binding in the nucleus. Upregulated circFAT1(e2) inhibits the growth of gastric cancer by suppressing the expression of three targeted genes of YBX1 (epidermal growth factor receptor, MET proto-oncogene and cell division cycle $25 \mathrm{~A}$ ).

Results from these studies suggest that the stability of circRNA-protein interaction might act as 'scaffolding molecules' in gastric cancer. Consequently, circRNAs acting as scaffolding molecules for protein complexes and network functional modules can modulate protein-protein interactions. circRNAs might work as sequence targeting elements, affecting the function of downstream target genes by simultaneously binding to RBPs.

circRNAs control the proliferation and progression of gastric cancer cells via regulating cellular metabolism. Cell glycolytic activity, including in aerobic conditions, is more active in cancer cells compared with normal cells, process known as the Warburg effect (63). In addition, tumor cells exhibit abnormal fatty acid and amino acid metabolism (64). Recent studies demonstrated that circRNAs are closely related to tumor metabolism and these are able to regulate gastric cancer metabolism through miRNA (65-67).

Upregulated circNRIP1 expression promotes the proliferation, invasion and migration of gastric cancer through activating AKT1/mTOR cell pathway by sponging miR-149-5p (Fig. 2) (65). circNRIP1 promotes anabolic activities and prevents metabolic decomposition by maintaining energy homeostasis, suggesting that circNRIP1 plays a crucial role in the regulation of tumor metabolism $(66,67)$.

In gastric cancer, ciRS-133 regulates substance metabolism and organic metabolism through PR domain-containing 16(PRDM16), enhancing the Warburg effect. ciRS-133 accelerates oxygen consumption and glucose consumption of brown fat cells, promoting white adipose tissue (WAT) burning through enhancing the expression of PRDM16 by adsorbing miR-133 in gastric cancer cells (Fig. 2) (68). Consequently, such effects are known to cause weight loss and systemic inflammation in patients with poor prognosis. Moreover, knockdown of ciRS-133 reduces cancer cachexia, decreasing oxygen consumption and glucose expenditure in mouse animal models (68). Thus, circRNAs contribute to cancer-associated cachexia and metabolic disorder by sponging miRNA to regulate WAT browning.

\section{4. circRNAs have a potential clinical value in gastric cancer}

Potential application of circRNAs in the diagnosis of gastric cancer. Several studies have demonstrated that circRNAs are differentially expressed in gastric cancer, as summarized in Table I. circRNAs are widely present in blood and gastric juice, and may have potential value for diagnosis (9-12). Therefore, circRNAs may work as novel biomarker-based screening tools for gastric cancer diagnosis and evaluation.

In gastric cancer, circ_102958 is closely associated to tumor, node, metastasis (TNM) cancer staging, but inversely associated to age, gender, tumor diameter and differentiation degree in lymph node metastasis (69). In addition, receiver operating characteristic curve analysis indicated that circ_102958 has a high diagnostic value. Together, these features suggested that circ_102958 may be a biomarker for early diagnosis of gastric cancer.

Owing to the lack of clinical symptoms and detection methods at the early stages of gastric cancer, the analysis of gastric juice could be advantageous for the detection of this type of cancer (70). For instance, circ_0014717 is downregulated in gastric juice of chronic atrophic gastritis. Individuals with chronic atrophic gastritis are at high risk of gastric cancer (71). This implies that circ_0014717 may be used for early screening of gastric cancer (72). In addition, circ_0000181 is downregulated in the plasma of patients with gastric cancer and is positively associated with the differentiation of gastric cancer cells and the level of carcinoembryonic antigen (CEA), which is a well-known gastric cancer biomarker (73). In addition, the specificity and sensitivity of hsa_circ_0000181 is higher than CEA and carbohydrate antigen19-9 in screening gastric cancer, suggesting that hsa_circ_0000181 may be a reliable plasma-based biomarker (74).

circRNAs as a prognosis biomarker in gastric cancer. circRNAs can potentially be used as a biomarker for the prognosis and the evaluation of treatment efficacy in gastric cancer. It has been demonstrated that circ_0000467 is highly expressed in tissue and plasma samples of patients with gastric cancer associated with a poor prognosis. Cox multivariate analysis showed that circ_0000467 might be an ideal independent prognostic factor (75). In addition, a previous study reported that the determination of prognostic factors in gastric cancer using circ_0000467 along with TNM stage is more accurate compared to that using TNM stage alone (75).

The expression of circ_KIAA1244 in plasma of patients with gastric cancer is lower than that in healthy patients without gastric cancer, which is inversely associated with TNM stage and lymph node metastasis, as well as a shorter overall survival. This is supported by univariate and multivariate analysis, which demonstrated that the expression of circ_KIAA1244 may be used as an independent prognostic indicator of the overall survival in patients with gastric cancer (76). 


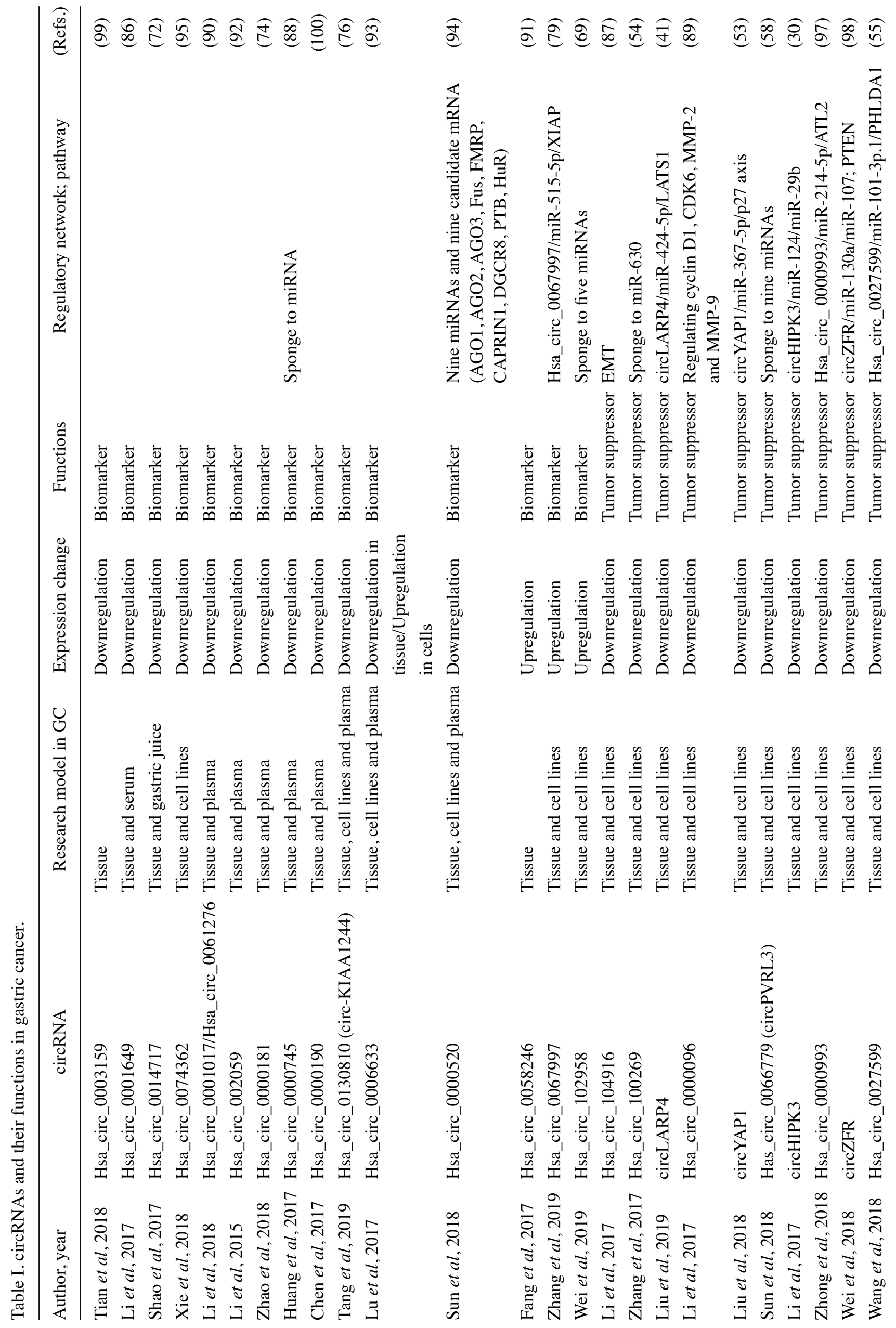


circRNA as a therapeutic target biomarker in gastric cancer. More recently, with the development of molecular biology and in-depth pathogenesis studies, the mechanistic function of circRNAs in metabolic and signal transduction pathways has also been investigated for gastric cancer. ciRS-7 possesses about 70 binding sites for miR-7 and has significant functions in several types of tumors, including gastric cancer, where it is highly expressed (77). ciRS-7 is able to enhance proliferation and migration of gastric cancer cells by upregulating PTEN/PI3K/AKT signaling pathway through sponging of miR-7 (78). Overexpression of ciRS-7 is also shown to promote the growth of xenograft tumors in vivo exhibiting a promising therapeutic value for gastric cancer (78). Therefore, developing inhibitors that can efficiently target ciRS-7 may be an important approach for therapy.

Highly expressed circ_0067997 promotes tumor growth in gastric cancer by sponging miR-515-5p, which results in the regulation of $x$-linked inhibitor of apoptosis (XIAP) expression $(79,80)$. Suppressing circ_0067997 by siRNA blocks a variety of apoptosis-related pathways and reduces cell viability, colony formation, proliferation and invasion of gastric cancer cell lines in vitro. circ_0067997 siRNAs treated MGC-803 cells inhibits the growth of tumors in vivo compared with si-NC treated cells, also suggesting a potential therapeutic target (81).

Finally, using Gene Ontology functional term and Kyoto Encyclopedia of Genes and Genomes signaling pathway analyses, gastric cancer was found to be linked to circRNAs in several important physiological processes, such as extracellular matrix degradation, binding to cell adhesion molecules and reaction with transforming growth factor $\beta$ (82).

Data from these studies demonstrated that circRNAs may contribute to the occurrence and development of gastric cancer, and its application in tumor therapy presents a valuable prospect. circRNAs may also be used as a therapeutic target for inhibiting tumor proliferation and metastasis.

\section{Future prospects}

Increasing evidence demonstrates that the expression of circRNAs affect the development and progression of gastric cancer and may exhibit great potential in diagnosis, prognosis and treatment of gastric cancer. Research within the mechanistic function of circRNAs is an emerging scientific field with an enormous potential. The differential expression of circRNAs in gastric cancer compared to non-cancer samples enables the use of these circRNAs as potential biomarkers $(41,83,84)$. circRNAs are have a stable structure and are widely expressed in various body fluids, including blood, saliva and gastric fluid (9-12). In addition, RNA sequencing and reverse transcription-quantitative PCR have made the detection of circRNAs more convenient and accurate than proteins as detection time is shorter, smaller amount of sample is required and the sensitivity is higher. Numerous circRNAs are highly enriched in the blood compared with the corresponding linear RNAs. Moreover, the expression of circRNAs in cancer tissue is higher than in precancerous tissue derived exosomes (85), which is consistent with another study showing that the levels of circ-KIAA1244 in plasma 
and plasma exosomes are similar (76). Therefore, circRNAs in extracellular exosomes represent the ideal candidates as reliable biomarkers for prognosis and diagnosis in gastric cancer. However, at present, circRNAs can only be used in combination with other biomarkers for cancer diagnosis, and the clinical application of circRNAs still requires further investigation and optimization. Although there are many studies about circRNAs, there no current standardized methodology for detecting circRNAs. Also, to the best of our knowledge there is no detailed study about the application of circRNAs as biomarkers for malignant cells, particularly regarding cost and the specificity and sensitivity for detecting cancer cells

The biological functions of several circRNAs may be utilized to explore the underlying mechanism of circRNAs during the development of gastric cancer. Despite many specific circRNAs reportedly expressed in gastric cancer, the features of circRNAs, including their biogenesis, degradation, location and function, remain to be explored. Therefore, comprehensive studies are required to determine the function and molecular mechanisms of circRNAs in gastric cancer. Moreover, further understanding of the relationship between circRNAs and gastric cancer may reveal the complex regulatory networks of carcinogenesis and accelerate the clinical application of circRNAs in prognosis, diagnosis and therapy of gastric cancer.

\section{Conclusions}

In the present review, the biological functions and molecular mechanisms of circRNAs were examined, and the clinical values of circRNAs with an emphasis on gastric cancer were summarized. Although numerous circRNAs have been identified, only a small amount of functional circRNAs has been elucidated, and most studies on circRNAs have focused on their regulation of cancer development through interactions with miRNAs and RBPs. In the future, the mechanistic study of its roles in genomic and mRNA transcription needs to be further explored. As circRNAs are known to be stable in different body fluids, including blood, circRNAs may act as ideal biomarkers and therapeutic targets for gastric cancer.

\section{Acknowledgements}

Not applicable.

\section{Funding}

This study was supported by grants from Shanghai Tongren Hospital (grant no. 2019shtrxx09), The Project of Shanghai Municipal Commission of Health and Family Planning (grant no. 20174Y0231), The National Natural Science Foundation of China (grant no. 81672335), Shanghai Jiaotong University, Medical Professionals Cross Fund (grant no. YG2016ZD10) and SJTU Research Project Grants 2019, University of Sydney, Australia.

\section{Availability of data and materials}

Not applicable.

\section{Authors' contributions}

CY and GQ conceived the study and wrote the manuscript. LS generated the tables and diagrams. LM and SB revised the manuscript for important intellectual content. All authors have read and approved the manuscript.

\section{Ethics approval and consent to participate}

Not applicable.

\section{Patient consent for publication}

Not applicable.

\section{Competing interests}

The authors declare that they have no competing interests.

\section{References}

1. Bray F, Ferlay J, Soerjomataram I, Siegel RL, Torre LA and Jemal A: Global cancer statistics 2018: GLOBOCAN estimates of incidence and mortality worldwide for 36 cancers in 185 countries. CA Cancer J Clin 68: 394-424, 2018.

2. Zhu L, Qin J, Wang J, Guo T, Wang Z and Yang J: Early gastric cancer: Current advances of endoscopic diagnosis and treatment. Gastroenterol Res Pract 2016: 9638041, 2016.

3. Huang RJ, Choi AY, Truong CD, Yeh MM and Hwang JH: Diagnosis and management of gastric intestinal metaplasia: Current status and future directions. Gut Liver 15: 596-603, 2019.

4. Hentze MW and Preiss T: Circular RNAs: Splicing's enigma variations. EMBO J 32: 923-925, 2013.

5. Vo JN, Cieslik M, Zhang Y, Shukla S, Xiao L, Zhang Y, Wu YM, Dhanasekaran SM, Engelke CG, Cao X, et al: The landscape of circular RNA in cancer. Cell 176: 869-881, 2019.

6. Wilusz JE and Sharp PA: Molecular biology. A circuitous route to noncoding RNA. Science 340: 440-441, 2013.

7. Tay Y, Rinn J and Pandolfi PP: The multilayered complexity of ceRNA crosstalk and competition. Nature 505: 344-352, 2014.

8. Conn SJ, Pillman KA, Toubia J, Conn VM, Salmanidis M, Phillips CA, Roslan S, Schreiber AW, Gregory PA and Goodall GJ: The RNA binding protein quaking regulates formation of circRNAs. Cell 160: 1125-1134, 2015.

9. Broadbent KM, Broadbent JC, Ribacke U, Wirth D, Rinn JL and Sabeti PC: Strand-Specific RNA sequencing in Plasmodium falciparum malaria identifies developmentally regulated long non-coding RNA and circular RNA. BMC Genomics 16: 454, 2015.

10. Lu T, Cui L, Zhou Y, Zhu C, Fan D, Gong H, Zhao Q, Zhou C, Zhao Y, Lu D, et al: Transcriptome-Wide investigation of circular RNAs in rice. RNA 21: 2076-2087, 2015.

11. Lu Z, Filonov GS, Noto JJ, Schmidt CA, Hatkevich TL, Wen Y, Jaffrey SR and Matera AG: Metazoan tRNA introns generate stable circular RNAs in vivo. RNA 21: 1554-1565, 2015.

12. Schmidt CA, Noto JJ, Filonov GS and Matera AG: A method for expressing and imaging abundant, stable, circular RNAs in vivo using tRNA splicing. Methods Enzymol 572: 215-236, 2016.

13. Jin M, Shi C, Yang C, Liu J and Huang G: Upregulated CircRNA ARHGAP10 Predicts an unfavorable prognosis in NSCLC through regulation of the miR-150-5p/GLUT-1 axis. Mol Ther Nucleic Acids 18: 219-231, 2019.

14. Ye F, Gao G, Zou Y, Zheng S, Zhang L, Ou X, Xie X and Tang H: CircFBXW7 inhibits malignant progression by sponging miR-197-3p and encoding a 185-aa protein in triple-negative breast cancer. Mol Ther Nucleic Acids 18: 88-98, 2019.

15. Zhu K, Zhan H, Peng Y, Yang L, Gao Q, Jia H, Dai Z, Tang Z, Fan J and Zhou J: Plasma hsa_circ_0027089 is a diagnostic biomarker for hepatitis B virus-related hepatocellular carcinoma. Carcinogenesis 19: 154, 2019.

16. Chen J,Chen T,Zhu Y,Li Y,Zhang Y, Wang Y,LiX, Xie X, Wang J, Huang M, et al: CircPTN sponges miR-145-5p/miR-330-5p to promote proliferation and stemness in glioma. J Exp Clin Cancer Res 38: 398, 2019. 
17. Xu G, Chen Y, Fu M, Zang X, Cang M, Niu Y, Zhang W, Zhang Y, Mao Z, Shao M, et al: Circular RNA CCDC66 promotes gastric cancer progression by regulating c-Myc and TGF- $\beta$ signaling pathways. J Cancer 11: 2759-2768, 2020.

18. Song H, Xu Y, Xu T, Fan R, Jiang T, Cao M, Shi L and Song J: CircPIP5K1A activates KRT80 and PI3K/AKT pathway to promote gastric cancer development through sponging miR-671-5p. Biomed Pharmacother 126: 109941, 2020.

19. Zhang L, Chang X, Zhai T, Yu J, Wang W, Du A and Liu N: A novel circular RNA, circ-ATAD1, contributes to gastric cancer cell progression by targeting miR-140-3p/YY1/PCIF1 signaling axis. Biochem Biophys Res Commun 14: 841-849, 2020.

20. Hu K, Qin X, Shao Y, Zhou Y, Ye G and Xu S: Circular RNA MTO1 suppresses tumorigenesis of gastric carcinoma by sponging miR-3200-5p and targeting PEBP1. Mol Cell Probes 56: $101562,2020$.

21. Ren S, Liu J, Feng Y, Li Z, He L, Li L, Cao X, Wang Z and Zhang Y: Knockdown of circDENND4C inhibits glycolysis, migration and invasion by up-regulating $\mathrm{miR}-200 \mathrm{~b} / \mathrm{c}$ in breast cancer under hypoxia. J Exp Clin Cancer Res 38: 388, 2019.

22. Liu Q, Zhang W, Wu Z, Liu H, Hu H, Shi H, Li S and Zhang X Construction of a circular RNA-microRNA-messengerRNA regulatory network in stomach adenocarcinoma. J Cell Biochem 12: 1317-1331, 2019.

23. Bi J, Liu H, Dong W, Xie W, He Q, Cai Z, Huang J and Lin T: Circular RNA circ-ZKSCAN1 inhibits bladder cancer progression through miR-1178-3p/p21 axis and acts as a prognostic factor of recurrence. Mol Cancer 18: 133, 2019.

24. Huang H, Wei L, Qin T, Yang N, Li Z and Xu Z: Circular RNA ciRS-7 triggers the migration and invasion of esophageal squamous cell carcinoma via miR-7/KLF4 and NF- $\kappa \mathrm{B}$ signals. Cancer Biol Ther 20: 73-80, 2019.

25. Hansen TB, Jensen TI, Clausen BH, Bramsen JB, Finsen B, Damgaard CK and Kjems J: Natural RNA circles function as efficient microRNA sponges. Nature 495: 384-388, 2013.

26. Chung DJ, Wang CJ, Yeh CW and Tseng TH: Inhibition of the proliferation and invasion of C6 glioma cells by tricin via the upregulation of focal-adhesion-kinase-targeting MicroRNA-7. J Agric Food Chem 66: 6708-6716, 2018.

27. Pan M, Li M, You C, Zhao F, Guo M, Xu H, Li L, Wang L and Dou J: Inhibition of breast cancer growth via miR-7 suppressing ALDH1A3 activity concomitant with decreasing breast cancer stem cell subpopulation. J Cell Physiol 235: 1405-1416, 2019.

28. Ye T, Yang M, Huang D, Wang X, Xue B, Tian N, Xu X, Bao L, $\mathrm{Hu} \mathrm{H}, \mathrm{Lv} \mathrm{T}$ and Huang Y: MicroRNA-7 as a potential therapeutic target for aberrant $\mathrm{NF}-\kappa \mathrm{B}$-driven distant metastasis of gastric cancer. J Exp Clin Cancer Res 38: 55, 2019

29. Ling Y, Cao C, Li S, Qiu M, Shen G, Chen Z, Yao F and Chen W: TRIP6, as a target of miR-7, regulates the proliferation and metastasis of colorectal cancer cells. Biochem Biophys Res Commun 514: 231-238, 2019.

30. Li Y, Zheng F, Xiao X, Xie F, Tao D, Huang C, Liu D, Wang M, Wang L, Zeng F and Jiang G: CircHIPK3 sponges miR-558 to suppress heparanase expression in bladder cancer cells. EMBO Rep 18: 1646-1659, 2017.

31. Xie H, Ren X, Xin S, Lan X, Lu G, Lin Y, Yang S, Zeng Z, Liao W, Ding YQ and Liang L: Emerging roles of circRNA_001569 targeting miR-145 in the proliferation and invasion of colorectal cancer. Oncotarget 7: 26680-26691, 2016.

32. Meng Q, Li S, Liu Y, Zhang S, Jin J, Zhang Y, Guo C, Liu B and Sun Y: Circular RNA circSCAF11 accelerates the glioma tumorigenesis through the miR-421/SP1/VEGFA axis. Mol Ther Nucleic Acids 17: 669-677, 2019.

33. Yan Y, Fu G, Ye Y and Ming L: Exosomes participate in the carcinogenesis and the malignant behavior of gastric cancer. Scand J Gastroenterol 52: 499-504, 2017.

34. Thomas LF and Sætrom P: Circular RNAs are depleted of polymorphisms at microRNA binding sites. Bioinformatics 30 : 2243-2246, 2014.

35. Zhao ZJ and Shen J: Circular RNA participates in the carcinogenesis and the malignant behavior of cancer. RNA Biol 14 514-521, 2017.

36. Lu J, Wang YH, Huang XY, Xie JW, Wang JB, Lin JX, Chen QY, Cao LL, Huang CM, Zheng CH and Li P: Circ-CEP85L suppresses the proliferation and invasion of gastric cancer by regulating NFKBIA expression via miR-942-5p. J Cell Physiol 5: 10, 2020.

37. Li H, Wei X, Yang J, Dong D, Hao D, Huang Y, Lan X, Plath M, Lei C, Ma Y, et al: CircFGFR4 promotes differentiation of myoblasts via binding miR-107 to relieve its inhibition of Wnt3a. Mol Ther Nucleic Acids 11: 272-283, 2018.
38. Ashwal-Fluss R, Meyer M, Pamudurti NR, Ivanov A, Bartok O, Hanan M, Evantal N, Memczak S, Rajewsky N and Kadener S: Circrna biogenesis competes with pre-mRNA splicing. Mol Cell 56: 55-66, 2014

39. Zheng J, Liu X, Xue Y, Gong W, Ma J, Xi Z, Que Z and Liu Y: TTBK 2 circular RNA promotes glioma malignancy by regulating miR-217/HNF1 $\beta /$ Derlin-1 pathway. J Hematol Oncol 10: 52, 2017.

40. Yang ZG, Awan FM, Du WW, Zeng Y, Lyu J, Wu, Gupta S, Yang W and Yang BB: The Circular RNA interacts with stat3, increasing its nuclear translocation and wound repair by modulating Dnmt3a and miR-17 function. Mol Ther 25: 2062-2074, 2017.

41. Liu KS, Pan F, Mao XD, Liu C and Chen J: Biological functions of circular RNAs and their roles in occurrence of reproduction and gynecological diseases. Am J Transl Res 11: 1-15, 2019.

42. Zhang Y, Liang W, Zhang P, Chen J, Qian H, Zhang X and Xu W: Circular RNAs: Emerging cancer biomarkers and targets. J Exp Clin Cancer Res 36: 152, 2017.

43. Pamudurti NR, Bartok O, Jens M, Ashwal-Fluss R, Stottmeister C, Ruhe L,Hanan M, WylerE, Perez-Hernandez D, Ramberger E, et al: Translation of CircRNAs. Mol Cell 66: 9-21, 2017.

44. Yang Y, Fan X, Mao M, Song X, Wu P, Zhang Y, Jin Y, Yang Y, Chen LL, Wang Y, et al: Extensive translation of circular RNAs driven by N(6)-methyladenosine. Cell Res 27: 626-641, 2017.

45. Burd CE, Jeck WR, Liu Y, Sanoff HK, Wang Z and Sharpless NE: Expression of linear and novel circular forms of an INK4/ARF-associated non-coding RNA correlates with atherosclerosis risk. PLoS Genet 6: e1001233, 2010.

46. Abdelmohsen K, Panda AC, Munk R, Grammatikakis I, Dudekula DB, De S, Kim J, Noh JH, Kim KM, Martindale JL and Gorospe M: Identification of HuR target circular RNAs uncovers suppression of PABPN1 translation by CircPABPN1. RNA Biol 14: 361-369, 2017.

47. Wang Y and Wang Z: Efficient backsplicing produces translatablecircular mRNAs. RNA 21: 172-179, 2015.

48. Li Z, Huang C, Bao C, Chen L, Lin M, Wang X, Zhong G, Yu B, $\mathrm{Hu}$ W, Dai L, et al: Exon-Intron circular RNAs regulate transcription in the nucleus. Nat Struct Mol Biol 22: 256-264, 2015.

49. Li F, Ma K, Sun M and Shi S: Identification of the tumor-suppressive function of circular RNA ITCH in glioma cells through sponging miR-214 and promoting linear ITCH expression. Am J Transl Res 10: 1373-1386, 2018.

50. Ouyang Y, Li Y, Huang Y, Li X, Zhu Y, Long Y, Wang Y, Guo X and Gong K: CircRNA circPDSS1 promotes the gastric cancer progression by sponging miR-186-5p and modulating NEK2. J Cell Physiol 234: 10458-10469, 2019.

51. Fry AM, O'Regan L, Sabir SR and Bayliss R: Cell cycle regulation by the NEK family of protein kinases. J Cell Sci 125: 4423-4433, 2012.

52. Chen J, Li Y, Zheng Q, Bao C, He J, Chen B, Lyu D, Zheng B, $\mathrm{Xu} Y$, Long Z, et al: Circular RNA profile identifies circPVT1 as a proliferative factor and prognostic marker in gastric cancer. Cancer Lett 388: 208-219, 2017.

53. Liu H, Liu Y, Bian Z, Zhang J, Zhang R, Chen X, Huang Y, Wang Y and Zhu J: Circular RNA YAP1 inhibits the proliferation and invasion of gastric cancer cells by regulating the miR-367-5p/p27 (Kip1) axis. Mol Cancer 17: 151, 2018.

54. Zhang Y, Liu H, Li W, Yu J, Li J, Shen Z, Ye G, Qi X and Li G: CircRNA_100269 is downregulated in gastric cancer and suppresses tumor cell growth by targeting miR-630. Aging (Albany NY) 9: 1585-1594,2017.

55. Wang L, Shen J and Jiang YL: Circ 0027599/PHDLA1 suppresses gastric cancer progression by sponging miR-101-3p.1. Cell Biosci 8: 58, 2018.

56. Yin QF, Yang L, Zhang Y, Xiang JF, Wu YW, Carmichael GG and Chen LL: Long noncoding RNAs with snoRNA ends. Mol Cell 48: 219-230, 2012.

57. Han B, Chao J and Yao H: Circular RNA and its mechanisms in disease: From the bench to the clinic. Pharmacol Ther 187: $31-44,2018$

58. Sun HD, Xu ZP, Sun ZQ, Zhu B, Wang Q, Zhou J, Jin H, Zhao A, Tang WW and Cao XF: Down-Regulation of circPVRL3 promotes the proliferation and migration of gastric cancer cells. Sci Rep 8: 10111, 2018.

59. Zhang M, Zhao K, Xu X, Yang Y, Yan S, Wei P, Liu H, Xu J, Xiao F, Zhou H, et al: A peptide encoded by circular form of LINC-PINT suppresses oncogenic transcriptional elongation in glioblastoma. Nat Commun 9: 4475, 2018.

60. Huang $\mathrm{H}$, Weng $\mathrm{H}$ and Chen $\mathrm{J}$ : $\mathrm{m}^{6} \mathrm{~A}$ Modification in coding and non-coding RNAs: Roles and therapeutic implications in cancer. Cancer Cell 37: 270-288, 2020. 
61. Wu P, Mo Y, Peng M, Tang T, Zhong Y, Deng X, Xiong F, Guo C $\mathrm{Wu}$ X, Li Y, et al: Emerging role of tumor-related functional peptides encoded by lncRNA and circRNA. Mol Cancer 19: 22, 2020.

62. Fang J, Hong H, Xue X, Zhu X, Jiang L, Qin M, Liang H and Gao L: A novel circular RNA, circFAT1(e2), inhibits gastric cancer progression by targeting miR-548g in the cytoplasm and interacting with YBX1 in the nucleus. Cancer Lett 442: 222-232, 2019.

63. Warburg $\mathrm{O}$, Wind $\mathrm{F}$ and Negelein E: The metabolism of tumors in the body. J Gen Physiol 8: 519-530, 1927.

64. Kimmelman AC and White E: Autophagy and tumor metabolism. Cell Metab 25: 1037-1043, 2017.

65. Zhang X, Wang S, Wang H, Cao J, Huang X, Chen Z, Xu P, Sun G, Xu J, Lv J and Xu Z: Circular RNA circNRIP1 acts as a microRNA-149-5p sponge to promote gastric cancer progression via the AKT1/mTOR pathway. Mol Cancer 18: 20, 2019.

66. Heras-Sandoval D, Perez-Rojas JM, Hernandez-Damian J and Pedraza-Chaverri J: The role of PI3K/AKT/mTOR pathway in the modulation of autophagy and the clearance of protein aggregates in neurodegeneration. Cell Signal 26: 2694-2701, 2014.

67. Wu ZH, Lin C, Liu CC, Jiang WW, Huang MZ, Liu X and Guo WJ: MiR-616-3p promotes angiogenesis and EMT in gastric cancer via the PTEN/AKT/mTOR pathway. Biochem Biophys Res Commun 501: 1068-1073, 2018

68. Zhang H, Zhu L, Bai M, Liu Y, Zhan Y, Deng T, Yang H, Sun W, Wang X, Zhu K, et al: Exosomal circRNA derived from gastric tumor promotes white adipose browning by targeting the miR-133/PRDM16 pathway. Int J Cancer 144: 2501-2515, 2019.

69. Wei J, Wei W, Xu H, Wang Z, Gao W, Wang T, Zheng Q, Shu Y and De W: Circular RNA hsa_circRNA 102958 may serve as a diagnostic marker for gastric cancer. Cancer Biomark 27 139-145, 2019.

70. Shao Y, Ye M, Li Q, Sun W, Ye G, Zhang X, Yang Y, Xiao B and Guo J: LncRNA-RMRP promotes carcinogenesis by acting as a miR-206 sponge and is used as a novel biomarker for gastric cancer. Oncotarget 7: 37812-37824, 2016.

71. Park YM, Kim JK, Baik SJ, Park JJ, Youn YH and Park H: Clinical risk assessment for gastric cancer in asymptomatic population after a health check-up: An individualized consideration of the risk factors. Medicine (Baltimore) 95: e5351, 2016.

72. Shao Y, Li J, Lu R, Li T, Yang Y, Xiao B and Guo J: Global circular RNA expression profile of human gastric cancer and its clinical significance. Cancer Med 6: 1173-1180, 2017.

73. Duffy MJ, Lamerz R, Haglund C, Nicolini A, Kalousová M, Holubec L and Sturgeon C: Tumor markers in colorectal cancer, gastric cancer and gastrointestinal stromal cancers: European group on tumor markers 2014 guidelines update. Int J Cancer 134: 2513-2522, 2014

74. Zhao Q, Chen S, Li T, Xiao B and Zhang X: Clinical values of circular RNA 0000181 in the screening of gastric cancer. J Clin Lab Anal 32: e22333, 2018.

75. Lu J, Zhang PY, Xie JW, Wang JB, Lin JX, Chen QY, Cao LL, Huang CM, Li P and Zheng CH: Hsa_circ_0000467 promotes cancer progression and serves as a diagnostic and prognostic biomarker for gastric cancer. J Clin Lab Anal 33: e22726, 2019.

76. Tang W, Fu K, Sun H, Rong D, Wang H and Cao H: CircRNA microarray profiling identifies a novel circulating biomarker for detection of gastric cancer. Mol Cancer 17: 137, 2018

77. Yang W, Gu J, Wang X, Wang Y, Feng M, Zhou D, Guo J and Zhou M: Inhibition of circular RNA CDR las increases chemosensitivity of 5-FU-resistant BC cells through up-regulating miR-7. J Cell Mol Med 23: 3166-3177, 2019.

78. Pan H, Li T, Jiang Y, Pan C, Ding Y, Huang Z, Yu H and Kong D: Overexpression of circular RNA ciRS-7 abrogates the tumor suppressive effect of miR-7 on gastric cancer via PTEN/PI3K/AKT signaling pathway. J Cell Biochem 119: 440-446, 2018

79. Zhang H, Wang X, Huang H, Wang Y, Zhang F and Wang S Hsa_circ_0067997 promotes the progression of gastric cancer by inhibition of miR-515-5p and activation of X chromosome-linked inhibitor of apoptosis (XIAP). Artif Cells Nanomed Biotechnol 47: 308-318, 2019.

80. Lencz T, Guha S, Liu C, Rosenfeld J, Mukherjee S, De Rosse P, John M, Cheng L, Zhang C, Badner JA, et al: Genome-Wide association study implicates NDST3 in schizophrenia and bipolar disorder. Nat Commun 4: 2739, 2013.

81. Sun J, Li J, Zhang W, Zhang J, Sun S, Li G, Song H and Wan D: MicroRNA-509-3p inhibits cancer cell proliferation and migration via upregulation of XIAP in gastric cancer cells. Oncol Res 25: 455-461, 2017.
82. Dang Y, Ouyang X, Zhang F, Wang K, Lin Y, Sun B, Wang Y, Wang $L$ and Huang Q: Circular RNAs expression profiles in human gastric cancer. Sci Rep 7: 9060, 2017.

83. Yang Y, Gao X, Zhang M, Yan S, Sun C, Xiao F, Huang N, Yang X, Zhao K, Zhou H, et al: Novel role of FBXW7 circular RNA in repressing glioma tumorigenesis. J Natl Cancer Inst 110: 3, 2018.

84.Zhou C, Molinie B, Daneshvar K, Pondick JV, Wang J, Van Wittenberghe N, Xing Y, Giallourakis CC and Mullen AC: Genome-Wide maps of m6A circRNAs identify widespread and cell-type-specific methylation patterns that are distinct from mRNAs. Cell Rep 20: 2262-2276, 2017.

85. Li Y, Zheng Q, Bao C, Li S, Guo W, Zhao J, Chen D, Gu J, He X and Huang S: Circular RNA is enriched and stable in exosomes: A promising biomarker for cancer diagnosis. Cell Res 25 981-984, 2015 .

86. Li WH, Song YC, Zhang H, Zhou ZJ, Xie X, Zeng QN, Guo K, Wang T, Xia P and Chang DM: Decreased expression of Hsa circ 00001649 in gastric cancer and its clinical significance. Dis Markers 2017: 4587698, 2017.

87. Li J, Zhen L, Zhang Y, Zhao L, Liu H, Cai D, Chen H, Yu J, Qi X and Li G: Circ-104916 is downregulated in gastric cancer and suppresses migration and invasion of gastric cancer cells. Onco Targets Ther 10: 3521-3529, 2017.

88. Huang M, He YR, Liang LC, Huang Q and Zhu ZQ: Circular RNA hsa_circ 0000745 may serve as a diagnostic marker for gastric cancer. World J Gastroenterol 23: 6330-6338, 2017.

89. Li P, Chen H, Chen S, Mo X, Li T, Xiao B, Yu R and Guo J: Circular RNA 0000096 affects cell growth and migration in gastric cancer. Br J Cancer 116: 626-633, 2017.

90. Li T, Shao Y, Fu L, Xie Y, Zhu L, Sun W, Yu R, Xiao B and Guo J: Plasma circular RNA profiling of patients with gastric cancer and their droplet digital RT-PCR detection. J Mol Med (Berl) 96: 85-96, 2018

91. Fang Y, Ma M, Wang J, Liu X and Wang Y: Circular RNAs play an important role in late-stage gastric cancer: Circular RNA expression profiles and bioinformatics analyses. Tumour Biol 39: 1393383814, 2017.

92.Li P, Chen S, Chen H, Mo X, Li T, Shao Y, Xiao B and Guo J: Using circular RNA as a novel type of biomarker in the screening of gastric cancer. Clin Chim Acta 444: 132-136, 2015

93. Lu R, Shao Y, Ye G, Xiao B and Guo J: Low expression of hsa_circ_0006633 in human gastric cancer and its clinical significances. Tumour Biol 39: 1393385489, 2017.

94. Sun H, Tang W, Rong D, Jin H, Fu K, Zhang W, Liu Z, Cao H and Cao X: Hsa circ_0000520, a potential new circular RNA biomarker, is involved in gastric carcinoma. Cancer Biomark 21: 299-306, 2018.

95. Xie Y, Shao Y, Sun W, Ye G, Zhang X, Xiao B and Guo J: Downregulated expression of hsa_circ_0074362 in gastric cancer and its potential diagnostic values. Biomark Med 12: 11-20, 2018

96. Rong D, Dong C, Fu K, Wang H, Tang W and Cao H Upregulation of circ_0066444 promotes the proliferation, invasion, and migration of gastric cancer cells. Onco Targets Ther 11: 2753-2761, 2018

97. Zhong S, Wang J, Hou J, Zhang Q, Xu H, Hu J, Zhao J and Feng J: Circular RNA hsa circ 0000993 inhibits metastasis of gastric cancer cells. Epigenomics 10: 1301-1313, 2018.

98. Wei H, Pan L, Tao D and Li R: Circular RNA circZFR contributes to papillary thyroid cancer cell proliferation and invasion by sponging miR-1261 and facilitating C8orf4 expression. Biochem Biophys Res Commun 503: 56-61, 2018.

99. Tian M, Chen R, Li T and Xiao B: Reduced expression of circRNA hsa_circ_0003159 in gastric cancer and its clinical significance. J Clin Lab Anal 32: 3, 2018.

100. Chen S, Li T, Zhao Q, Xiao B and Guo J: Using circular RNA hsa circ 0000190 as a new biomarker in the diagnosis of gastric cancer. Clin Chim Acta 466: 167-171, 2017.

101. Zhou LH, Yang YC, Zhang RY, Wang P, Pang MH and Liang LQ: CircRNA 0023642 promotes migration and invasion of gastric cancer cells by regulating EMT. Eur Rev Med Pharmacol Sci 22 2297-2303, 2018.

102. Sun H, Xi P, Sun Z, Wang Q, Zhu B, Zhou J, Jin H, Zheng W, Tang W, Cao H and Cao X: Circ-SFMBT2 promotes the proliferation of gastric cancer cells through sponging miR-182-5p to enhance CREB1 expression. Cancer Manag Res 10: 5725-5734, 2018.

103. Shen F, Liu P, Xu Z, Li N, Yi Z, Tie X, Zhang Y and Gao L: CircRNA_001569 promotes cell proliferation through absorbing miR-145 in gastric cancer. J Biochem 165: 27-36, 2019.

This work is licensed under a Creative Commons Attribution-NonCommercial-NoDerivatives 4.0 International (CC BY-NC-ND 4.0) License. 